\title{
SNPs and real-time quantitative PCR method for constitutional allelic copy number determination, the VPREB1 marker case
}

\author{
Marcello Frigerio ${ }^{1 *}$, Elena Passeri ${ }^{2,3}$, Tiziana de Filippis ${ }^{4}$, Daniela Rusconi ${ }^{6}$, Rea Valaperta ${ }^{1}$, Mario Carminati ${ }^{8}$, \\ Anita Donnangelo ${ }^{1}$, Elena Costa ${ }^{1}$, Luca Persani ${ }^{4,5}$, Palma Finelli ${ }^{6,7}$ and Sabrina Corbetta ${ }^{2,3}$
}

\begin{abstract}
Background: 22q11.2 microdeletion is responsible for the DiGeorge Syndrome, characterized by heart defects, psychiatric disorders, endocrine and immune alterations and a 1 in 4000 live birth prevalence. Real-time quantitative $P C R$ ( $(\mathrm{PCR})$ approaches for allelic copy number determination have recently been investigated in 22q11.2 microdeletions detection. The qPCR method was performed for 22q11.2 microdeletions detection as a firstlevel screening approach in a genetically unknown series of patients with congenital heart defects. A technical issue related to the VPREB1 qPCR marker was pointed out.
\end{abstract}

Methods: A set of 100 unrelated Italian patients with congenital heart defects were tested for $22 q 11.2$ microdeletions by a qPCR method using six different markers. Fluorescence In Situ Hybridization technique (FISH) was used for confirmation.

Results: qPCR identified six patients harbouring the 22q11.2 microdeletion, confirmed by FISH. The VPREB1 gene marker presented with a pattern consistent with hemideletion in one $3 \mathrm{Mb}$ deleted patient, suggestive for a long distal deletion, and in additional five non-deleted patients. The long distal 22q11.2 deletion was not confirmed by Comparative Genomic Hybridization. Indeed, the VPREB1 gene marker generated false positive results in association with the rs1320 G/A SNP, a polymorphism localized within the VPREB1 marker reverse primer sequence. Patients heterozygous for rs1320 SNP, showed a QPCR profile consistent with the presence of a hemideletion.

Conclusions: Though the GPCR technique showed advantages as a screening approach in terms of cost and time, the VPREB1 marker case revealed that single nucleotide polymorphisms can interfere with $\mathrm{QPCR}$ data generating erroneous allelic copy number interpretations.

Keywords: qRT-PCR allelic copy number, 22q11.2 microdeletion, DiGeorge Syndrome

\section{Background}

Real-time quantitative PCR (qPCR) performed with standard curves has been proposed as a routine, reliable and highly sensitive assay for gene expression analysis [1]. qPCR applications are as well becoming a reference method, alternative to Southern blot and Fluorescence In Situ Hybridization (FISH), for the measurement of gene copy number in human tumours with allelic imbalances [2]. Similar applications have been finally adopted

\footnotetext{
* Correspondence: marcello.frigerio@gmail.com

${ }^{1}$ Research Laboratories - Molecular Biology, IRCCS Policlinico San Donato, San Donato Milanese (MI), Italy

Full list of author information is available at the end of the article
}

for constitutional allelic copy number determination with several examples in the detection of 22q11.2 microdeletions responsible for the homonymous syndrome, also known as DiGeorge syndrome (DGS; OMIM 188400) [3-7]. DGS encompasses a wide spectrum of clinical features with variable expression including dysmorphic facies, palate abnormalities, congenital conotruncal cardiac defects, endocrine dysfunctions, T-cell mediated immune deficiency and psychiatric disorders [8]. This autosomal dominant disorder constitutes a breeding ground for the development of a real-time qPCR based technique because of its frequency (1:4000 live births) and its allelic heterogeneity. Indeed, the DGS

\section{Biomed Central}


clinical phenotype may result from multiple types of microalterations at the same locus including the most frequent $3 \mathrm{Mb}$ deletion and the smaller nested $1.5 \mathrm{Mb}$ deletion, occurring respectively in approximately $90 \%$ and $7 \%$ of patients with a $22 \mathrm{q} 11.2$ anomaly, and other atypical and rare microdeletions and microduplications affecting the critical $3 \mathrm{Mb}$ region and its proximal and distal flanking regions [9].

The specific qPCR analysis for the $22 \mathrm{q} 11.2$ region allelic copy number determination performed with SYBR Green chemistry has been described by Weksberg [6]. It based on the comparison among the amplifications of 10 different markers localized within the deletion critical 22q11.2 sequence and its proximal regions affected with rare recently described distal deletions [9]. As the SYBR Green molecule is a fluorescent non-specific intercalant agent, the primers design represents the essential point for the method specificity. The GPCR primers were designed within regions of unique sequence avoiding the complex repetitive regions, possibly within gene exons sequences [6,7].

The qPCR method was previously proposed as a sensitive technique for precise deletion breakpoints definition with theoretical useful applications in genotype-phenotype correlation studies [6,7]. Indeed, the qPCR approach could be applied to patients with an evident syndromic phenotype who are not diagnosed with clinical standard cytogenetic methods due to atypical alterations. Considering its intrinsic technical advantages, we tested the Weksberg qPCR method for 22q11.2 microdeletion detection as a first-level screening technique exploitable for wide population studies and useful as screening approach to the high-resolution cytogenetic tools such as Comparative Genomic Hybridization (CGH) arrays.

In the present study the Weksberg's qPCR approach for 22q11.2 microdeletion detection was performed in a wide genetically unknown series characterized by a phenotypic trait (congenital heart defects) included in DGS clinical features. A technical issue related to the VPREB1 qPCR marker was pointed out.

\section{Results}

\section{Real-time quantitative $P C R$ results}

Eighty-eight patients presented a qPCR markers profile similar to that observed in del22q11.2 negative control samples indicating no loss in allelic copy number.

Six patients, five unrelated and a homozygotic twin, harboured a microdeletion as they showed a qPCR markers combination suggestive of a long distal deletion. In these patients D22S181 and VPREB1 amplifications were consistent with the presence of two allelic copies, whereas the amplification profile of PRODH, TUPLE1, COMT and D22S936 indicated a loss of one allelic copy.
Presence of microdeletions was confirmed in all the six patients with a standard FISH analysis.

An unexpected result from the patients screening was the finding in five patients of qPCR marker profiles characterized by the only VPREB1 hemideletion, suggestive for the presence of atypical distal deletions. One further patient (patient no.4 in Table 1; index case) showed a qPCR set with hemideletion in the PRODH, TUPLE1, COMT, D22S936 and VPREB1 markers that could be consistent with a long atypical deletion.

VPREB1 marker standard curves and melting curves analysis performed in all subjects presenting the VPREB1 marker hemideletion was consistent with the 10 del22q11.2 negative controls profile thus indicating no difference in primer efficiency and amplicon specificity.

\section{Fluorescence in situ hybridization and Array CGH on index case DNA}

Standard FISH analysis confirmed the presence of a microdeletion in the 22q11 region in the index case. The patient's DNA was further investigated by CGH assay to test the qPCR-based deletion extension hypothesis.

Array CGH revealed a heterozygous deletion on chromosome 22q11.21 spanning at least $2.56 \mathrm{Mb}$ (chr22: 17,274,835-19,835,417, hg18; chr22: 18,894,83521,505,417, hg19 UCSC) (Figure 1). The centromeric breakpoint maps between probe A_16_P41477672 and probe A_16_P41477688 (Chr22: 17,270,271-17,274,894 bp, UCSC, hg18; chr22: 18,890,271-18,894,894, hg19 UCSC) in LCR22-2, whereas the distal breakpoint maps between probe A_16_P41484416 and probe A_16_P03603092 (Chr22: 19,835,358-19,891,514 bp, UCSC, hg18; chr22: 21,505,358-21,561,514, hg19 UCSC) in LCR22-4. CGH assay confirmed the presence of the classical deletion. Nor the critical region distal probes nor the specific VPREB1 probe (Figure 2, line $\mathrm{D}$ ) of the $\mathrm{CGH}$-array showed the presence of a hemideletion in VPREB1 gene or in its proximal region.

\section{VPREB1 genotype results}

VPREB1 gene sequence analysis performed with Set 1 primers (Table 1 and Figure 2, line $C$ ) on the six subjects with hemideletion in VPREB1 marker showed the presence of heterozygosity for the rs1320 A/G SNP, a rare (minor allele frequency in the European Population $=0.062$ ) missense single nucleotide polymorphism [10] localized within the complementary region of the qPCR $V P R E B 1$ marker reverse primer. This finding was consistent with the interpretation of the qPCR VPREB1 marker hemideletions as false positive results.

The subsequent sequencing with Set 2 primers (Table 2 and Figure 2, line C) of the remaining 94 subjects with a qPCR VPREB1 marker were consistent with 
Table 1 Overview of the qPCR markers results

\begin{tabular}{cccccccc}
\hline Patients & No. & \multicolumn{5}{c}{ 22q11.2 region qPCR markers results $\left(\Delta K_{\mathrm{t}}\right)$} \\
\hline & & D22S181 & PRODH & TUPLE1 & COMT & D22S936 & VPREB1 \\
\hline Not deleted & 88 & $0.007 \pm 0.123$ & $-0.009 \pm 0.006$ & $-0.018 \pm 0.076$ & $0.039 \pm 0.009$ & $-0.002 \pm 0.062$ & $0.082 \pm 0.008$ \\
22q11.2 & 6 & $-0.004 \pm 0.140$ & $-0.982 \pm 0.039$ & $-1.076 \pm 0.014$ & $-0.958 \pm 0.154$ & $-0.887 \pm 0.047$ & $0.080 \pm 0.024$ \\
hemideletion & & & & & & \\
$\quad$ Patient no.4 & 1 & $0.106 \pm 0.014$ & $-0.992 \pm 0.025$ & $-1.037 \pm 0.069$ & $-0.994 \pm 0.104$ & $-0.918 \pm 0.001$ & $-1.050 \pm 0.186$ \\
VPREB1 hemideletion & 5 & $0.067 \pm 0.087$ & $0.005 \pm 0.098$ & $0.038 \pm 0.095$ & $0.005 \pm 0.115$ & $0.020 \pm 0.110$ & $-1.093 \pm 0.042$ \\
\hline
\end{tabular}

Data are expressed as the mean $\Delta K C_{t} \pm$ standard deviation calculated on all the subjects of each patients set. $\Delta K C_{t}$ consistent with a loss of one allelic copy are indicated in bold.

the presence of two allelic copies demonstrating that all patients were homozygous carriers of the rs 1320 ancestral $\mathrm{G}$ allele.

No immunological disorder was found to be associated with the rs1320 A allele.

\section{Discussion}

The real-time quantitative PCR approach could represent a robust, fast and accurate assay for the detection of copy number alterations in genomic DNA [2-7]. Two peculiar advantages of the GPCR method have been focused: the detection of atypical microdeletions undiagnosed by diagnostic standard FISH approach and the accurate mapping of deletion breakpoints. Previous studies on the real-time quantitative application to 22q11.2 microdeletions detection [6,7] were performed in small series with a previous cytogenetic analysis positive for 22q11.2 microdeletions, as the main purpose was the refinement of the deletion breakpoints maps with the increasing of the number of markers in the 22q11.2 deleted region.

In the present study, we performed the qPCR method in a large paediatric genetically unknown patient cohort screening. We selected a reduced number of 22q11.2 region markers to provide a rapid patient genotype. The six markers effectively identified and differentiated the
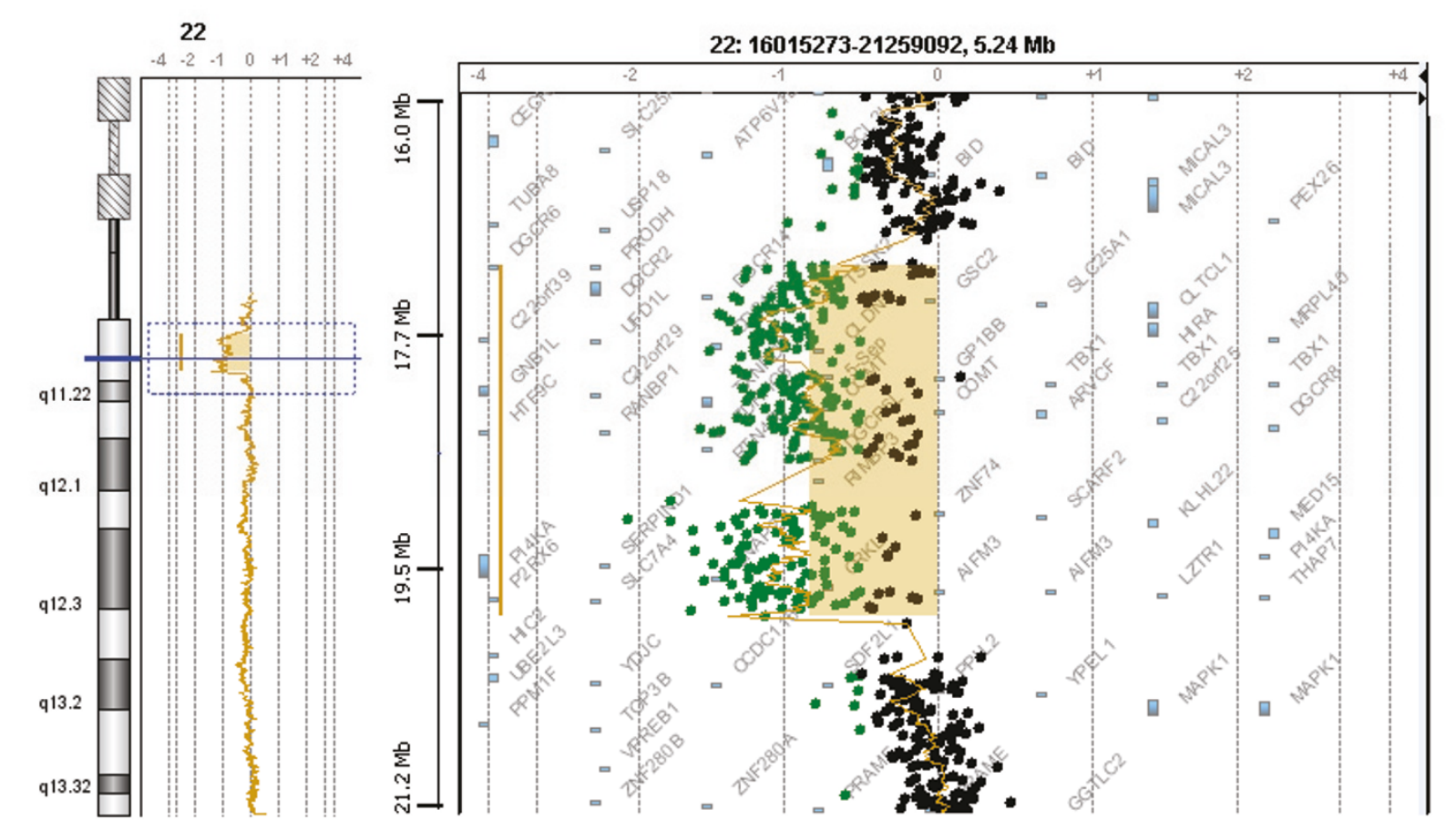

Figure 1 Array CGH profile of index case DNA. A) Whole chromosome 22 array profile is shown. Scattered plot analysis reveals a deletion in 22 q22.12 (horizontal shift to left of 0). B) Zoomed-in gene view of panel A which focuses on a $5.2 \mathrm{Mb}$ window within $22 \mathrm{q} 22.12 \mathrm{containing}$ the deletion. Each point represents a single probe. $\log _{2}$ (ratio) was plotted for all oligonucleotide probes based on their chromosome positions. Aberration calls identified by ADM-2 algorithm (coloured shaded areas) are shown. 


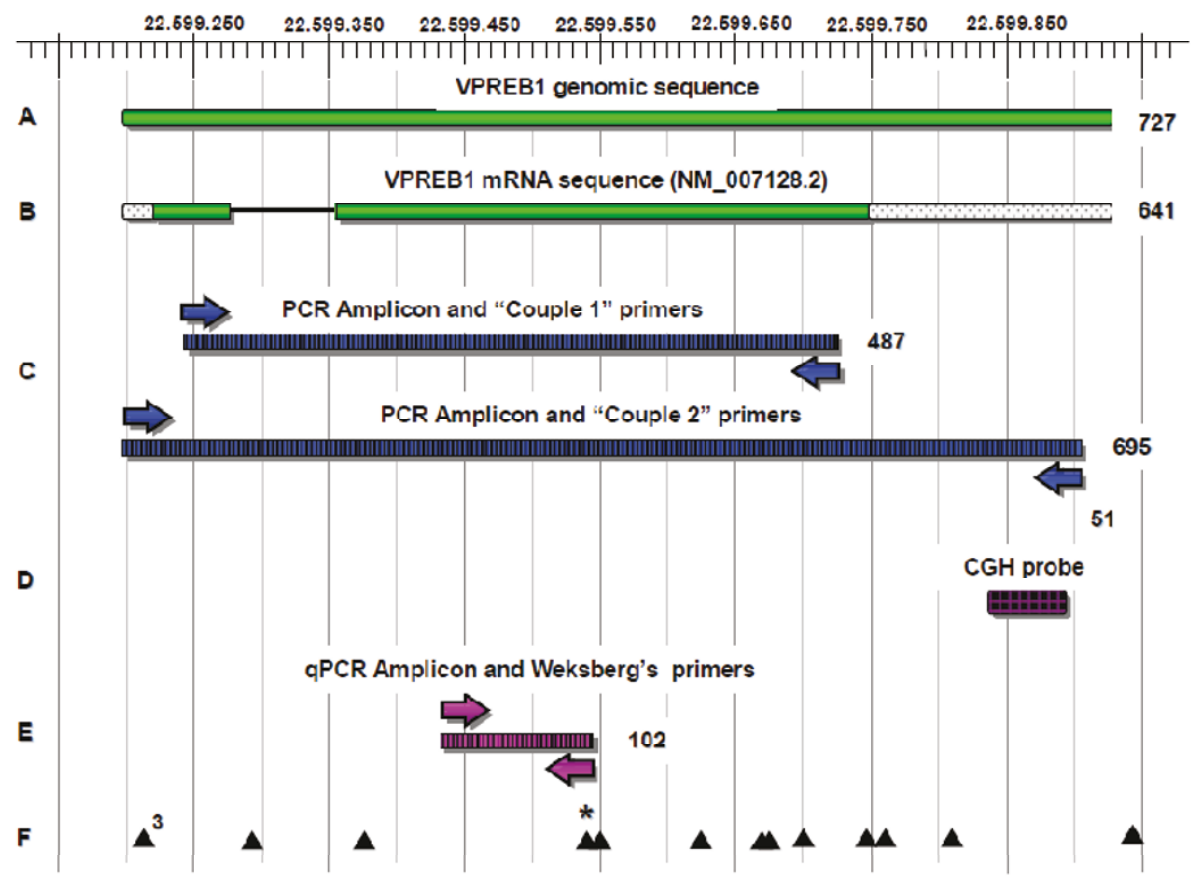

Figure 2 Schematics of the VPREB1 gene 22q11.2 region. A) Genomic sequence of VPREB1 gene. B) VPREB1 mRNA sequence (NM_007128.2), intron sequence was represented with a black line, dotted ends indicated untranslated regions. C) PCR products resulting from Set 1 and Set 2 primers amplification. D) CGH-array VPREB1 probe. E) VPREB1 Weksberg's primers and relative qPCR product. F) Locations of SNPs, the rs1320 polymorphism is highlighted with a $\left(^{*}\right)$. For $A-E$, the number on the right indicates the base pairs present in each relative segment.

typical 22q11.2 alterations (the $3 \mathrm{Mb}$ and the $1.5 \mathrm{Mb}$ microdeletions) and the rare atypical microdeletions. Our results confirmed the efficiency of the method in an unselected series of young patients with congenital heart defects, identifying six patients harbouring the classic $3 \mathrm{Mb}$ microdeletion fully concordant with the FISH analysis results. Interestingly, this approach provided DGS diagnosis in patients with atypical mild clinical presentations, extending the accuracy of the diagnosis and allowing genetic counselling.

At variance with the data presented by Weksberg et al. $[6,7]$, the VPREB1 qPCR marker analysis produced results suggestive for the presence of distal deletions at an unexpected high frequency. Nonetheless, the long

Table 2 Sequences and locations of VPREB1 PCR amplification primers

\begin{tabular}{cccc}
\hline $\begin{array}{c}\text { Primer } \\
\text { Sets }\end{array}$ & Primer Sequences & $\begin{array}{c}\text { Amplicon } \\
\text { Size }\end{array}$ & $\begin{array}{c}\text { Genomic Location of } \\
\text { Amplicon }\end{array}$ \\
\hline Set 1 & AGAGCTCTGCATGTCTGCAC & 695 & $\begin{array}{c}22599205-22599900 \\
\text { (Chr 22) }\end{array}$ \\
& TTCCCTAATGCAGTCTCCAA & & \\
Set 2 & TCCTGCTCATGCTGTTGTC & 487 & $\begin{array}{c}\text { 22599242-22599729 } \\
\text { (Chr 22) }\end{array}$ \\
& CTGCAGTGGGTCCATTCT & & \\
\hline
\end{tabular}

extension of the deletion was not confirmed by the $\mathrm{CGH}$ analysis. Investigating the molecular basis of these discrepancies, we considered that the VPREB1 gene sequence is characterized by a high frequency of SNPs and that the Weksberg's qPCR VPREB1 marker reverse primer sequence was specifically designed for the exon 2 VPREB1 sequence containing the rs1320 SNP G ancestral allele.

Our data suggested that the presence in heterozygosity of the rs1320 A allele constitutes a critical mismatch in the reverse primer sequence leading to an inefficient primer annealing and a consequent misamplification. Indeed, under these assumptions, patients with an heterozygous profile for rs1320, that are carriers of a single fully complementary allelic copy for qPCR VPREB1 marker amplification, should produce, as detected in our experience, a $\Delta \mathrm{KC}_{\mathrm{t}}$ value range similar to that owned by patients with a constitutional single allelic copy due to microdeletion. This hypothesis resembles similar experiences previously described $[11,12]$.

\section{Conclusions}

The VPREB1 qPCR marker case here described underscored the effect of a single nucleotide substitution in the primer complementary sequence that can lead to an 
invalid qPCR amplification resulting in a defective allelic copy number interpretation. From this observation we take advantage to stress the relevance of the primer design for any kind of real-time PCR using standard curves analysis. Primer sequences should be designed avoiding both the repeated sequence regions and the high polymorphic loci.

Finally, data here presented highlighted how this qPCR method could be a rapid, cost-effective and easy to reproduce assay for 22q11.2 region genotyping when applied with a confined number of markers on a highthroughput system. These advantages are desirable for an efficient first-level screening approach. We feel that the qPCR approach could represent a valid alternative to the more classical and expensive cytogenetic analysis, and therefore a helpful clinical tool for the 22q11 screening in patients with a non-classic phenotype.

\section{Methods}

\section{Subjects}

qPCR method was adopted for the screening of a retrospective cohort of 100 consecutive unrelated Italian paediatric patients [ 48 females, 52 males; 6.51 (4.48-9.06) years (median age, IQ range)] referred to the Paediatric Cardiac Surgery Unit of the IRCCS Policlinico San Donato between 2007 and 2009 for the management of congenital heart defects. The congenital heart defects were: atrial septal defects (28\%), ventricular septal defects (20\%), patent ductus arteriosus (15\%), aortic coartations (14\%), Tetralogy of Fallot (12\%), miscellaneous uncommon lesions (11\%). Patients affected with known genetic syndrome were excluded. The genetic study was adherent to the principles of Helsinki Declaration. Parents of all participants signed an informed consent for genetic testing.

\section{Real-time quantitative PCR (qPCR)}

Genomic DNA was extracted using standard procedures from EDTA-blood samples collected from patients at the time of admission after an overnight fasting. Realtime quantitative PCR analysis was performed with the D22S181, PRODH, TUPLE1, COMT, D22S936 and $V P R E B 1$ markers to obtain an immediate overview of the patient genotype (Figure 3). Primer sequences were derived from Weksberg studies [6,7]. Though the marker sets used in the present study were reduced from those reported by Weksberg [6,7], the efficient discrimination of the two most frequent alterations, the $3 \mathrm{Mb}$ and the $1.5 \mathrm{Mb}$ hemizygous deletions, and the detection of atypical deletions were ensured. G6PDH and HEM3 markers were also amplified for the subsequent data normalization.

Real-time qPCR assays were performed in a $25 \mu \mathrm{l}$ reaction volume containing $12.5 \mu \mathrm{l} 2 \times$ SYBR Green I PCR

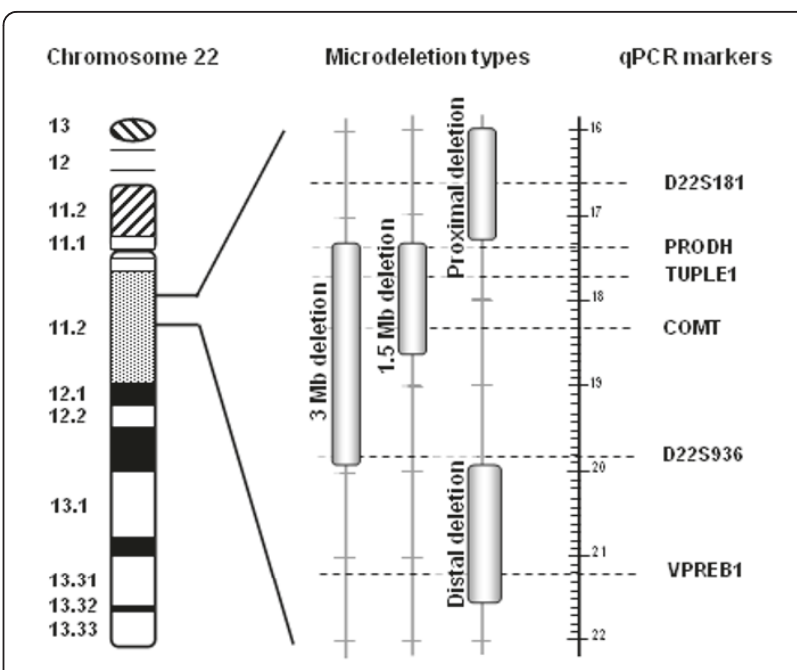

Figure 3 Representation of Chromosome 22 with enlargement of the 22q11.2 region and $q P C R$ markers locations. $q P C R$ markers localization was shown with respect to the two most classical $3 \mathrm{Mb}$ and $1.5 \mathrm{Mb}$ microdeletions and two different examples of atypical deletions. PRODH, TUPLE1 and COMT markers recognized $3 \mathrm{Mb}$ and 1,5 Mb microdeletions; D22S936 marker allowed to distinguish between $3 \mathrm{Mb}$ or $1.5 \mathrm{Mb}$ microdeletions; D22S181 and VPREB1, both localized outside the critical deletion region, allowed the detection of respectively proximal and distal atypical microalterations.

Master Mix (Applied Biosystems, Foster City, CA, USA), 10 ng genomic DNA, forward and reverse primers at final concentration of $800 \mathrm{nM}$ for the specific $22 \mathrm{q} 11.2$ region markers and $400 \mathrm{nM}$ for G6PDH and HEM3 markers. Reactions were performed in 96-well MicroAmp Optical Reaction Plates (Applied Biosystems) using the ABI Prism 7900 high-throughput sequence detection systems (Applied Biosystems) and data were processed by the associated SDS software version 2.3 (Applied Biosystems). Standard curves for all the primers set used were generated with series of $\log$ dilution of 4 del22q11.2 negative samples genomic DNA. Slopes derived from standard curves were used for data normalization following Weksberg's instructions [6]. Reaction specificity was confirmed with melting curves analysis and agarose gel electrophoresis experiments. Each qPCR assay included at least 3 del22q11.2 negative control samples; all reactions were performed in triplicate, with replicates being performed on different days. Allelic copy number of the 22q11.2 region markers where finally determined with the comparison of normalized data of control samples and patients. Final $\Delta \mathrm{KC}_{\mathrm{t}}$ values were considered as follows: the interval -0.75 to -1.25 indicated loss of one copy (presence of hemizygous microdeletion), whereas a ratio of $0.00 \pm 0.25$ corresponded to two allelic copies (no microdeletion). Data normalization and allelic copy number equations were derived from Weksberg's studies [6,7]. 
All qPCR markers presented at the standard curves analysis an amplification efficiency similar to that reported by Weksberg [6], however each standard curve slope value was found in the $3.32 \pm 0.25$ range allowing the successive amplification comparison and data elaboration. Melting curve analysis and agarose gel-electrophoresis assay confirmed the amplicons specificity showing respectively a single peak on the dissociation curve plot and a single band of the expected size on the electrophoretic run. qPCR results for this study series are summarized in Table 1.

\section{Fluorescence in situ hybridization analysis}

The FISH technique was performed on peripheral blood lymphocytes according to the manufacturer's instruction. A commercial probe results in the simultaneous labelling of the 22q11.2 chromosome region that includes TUPLE1 gene also known as HIRA gene (red labelling) and the terminal region of chromosome 22 as control (green labelling) was used. Twenty patient's metaphases were analyzed under a fluorescence microscope (Leica Microsystem, Wetzlar, Germany).

\section{Array $\mathrm{CGH}$ analysis}

For CGH, genomic DNA was extracted from proband's blood using the Genelute Blood Genomic DNA Kit (Sigma-Aldrich Corp. St. Louis, MO, USA) according to the manufacture's instructions. Pooled DNA from the peripheral blood of 10 healthy donors (Promega, Madison, WI), sex-matched to the sample was used as a reference for the DNA extracted from peripheral blood. Genomic DNA $(1.5 \mu \mathrm{g})$ was hybridized to the $244 \mathrm{~KB}$ microarray (Agilent Technologies, Palo Alto, CA, USA), consisting of $\sim 236,00060$-mer oligonucleotide probes covering the entire genome at the average spatial resolution of $\sim 30 \mathrm{~Kb}$, and processed according to the manufacturer's instructions.

The dye emission capture was performed by a duallaser Agilent Scanner. Images were extracted using Agilent Feature Extraction software 9.1 and analysed by DNA Analytics 4.0 software. A log ratio plot between test and reference genomic DNA was assigned such that aberrations in test DNA copy number at a particular locus are observed as the deviation of the ratio plots from a modal value of 0 ; aberration calls was identified by the ADM-2 algorithm.

\section{VPREB1 Sequencing}

VPREB1 amplification was performed on all qPCR analysed samples with the same genomic DNA template used in qPCR experiments. Two different primers sets were designed using Primer3 online software [13]. Set 1 amplicon performed the sequencing of the entire VPREB1 gene, while Set 2 amplicon was centred within the qPCR marker reverse primer sequence (Figure 2). Primer sequences are shown in Table 2.

PCR was performed in a $25 \mu \mathrm{l}$ reaction volume containing 200 ng genomic DNA, 2.5 U Platinum Taq DNA Polymerase (Invitrogen, Madison, WI, USA), $25 \mu \mathrm{mol}$ of each primer, $100 \mu \mathrm{mol} / \mathrm{L}$ of each dNTP, $1 \times$ PCR buffer (Invitrogen) and $1.5 \mathrm{mM} \mathrm{MgCl} 2$ with the use of a $\mathrm{MyCy}$ cler thermal cycler (Biorad, Hercules, CA, USA). Cycling variables were as follows: $94^{\circ} \mathrm{C}$ for 5 min (first denaturing step); 30 cycles of $94^{\circ} \mathrm{C}$ for $1 \mathrm{~min}, 56.3^{\circ} \mathrm{C}$ for $1 \mathrm{~min}$ and $72^{\circ} \mathrm{C}$ for $2 \mathrm{~min}$; and $72^{\circ} \mathrm{C}$ for $10 \mathrm{~min}$ (last extension step). We did bidirectional sequencing of the purified PCR products (GE Healthcare, Buckinghamshire, UK) by a BigDye terminator sequencing kit (Applied Biosystems) and a 3100 genetic analyser (Applied Biosystems). Sequences were analysed by the ABI Prism Sequencing Analysis Software (version 3.7, Applied Biosystems).

\section{Acknowledgements}

The study was supported by the University of Milan found PUR 2008, by IRCCS Policlinico San Donato Research Found and by a Ministry of Health grant "Ricerca Corrente" to Istituto Auxologico Italiano IRCCS.

\section{Author details}

${ }^{1}$ Research Laboratories - Molecular Biology, IRCCS Policlinico San Donato, San Donato Milanese (MI), Italy. ${ }^{2}$ Endocrinology and Diabetology Unit, IRCCS Policlinico San Donato, San Donato Milanese (MI), Italy. ${ }^{3}$ Department of Medical-Surgical Sciences, Università di Milano, San Donato Milanese (MI), Italy. ${ }^{4}$ Laboratorio di Ricerche Endocrino-Metaboliche, IRCCS Istituto Auxologico Italiano, Milan, Italy. ${ }^{5}$ Department of Medical Sciences, Università di Milano, Milan, Italy. ${ }^{6}$ Laboratory of Medical Cytogenetics and Molecular Genetics, IRCCS Istituto Auxologico Italiano, Cusano Milanino (MI), Italy. ${ }^{7}$ Department of Biology and Genetics for Medical Sciences, Università di Milano, Milan, Italy. ${ }^{8}$ Pediatric Cardiology and Adult with Congenital Heart Disease Department, IRCCS Policlinico San Donato, San Donato Milanese (MI), Italy.

\section{Authors' contributions}

MF conceived the study, participated in its design and coordination, carried out the real-time qPCR studies and drafted the manuscript. TF and LP participated in the VPREB1 gene sequencing. DR and PF carried out the cytogenetic study. RV and AD participated in molecular genetics studies and, together with EC, participated in the coordination of the study and helped to draft the manuscript. EP contributed with pertinent clinical information and, together with SC, contributed to overall study design. All authors read and approved the final manuscript.

\section{Competing interests}

The authors declare that they have no competing interests.

Received: 28 June 2010 Accepted: 5 May 2011 Published: 5 May 2011

\section{References}

1. VanGuilder HD, Vrana KE, Freeman WM: Twenty-five years of quantitative PCR for gene expression analysis. Biotechniques 2008, 44:619-626.

2. Hoebeeck J, Speleman F, Vandesompele J: Real-time quantitative PCR as an alternative to Southern blot or fluorescence in situ hybridization for detection of gene copy number changes. Methods Mol Biol 2007, 353:205-226.

3. Ivanov D, Kirov G, Norton N, Williams HJ, Williams NM, Nikolov I, Tzwetkova R, Stambolova SM, Murphy KC, Toncheva D, Thapar A, O'Donovan MC, Owen MJ: Chromosome 22q11 deletions, velo-cardiofacial syndrome and early-onset psychosis. Molecular genetic study. $\mathrm{Br} J$ Psychiatry 2003, 183:409-413. 
4. Kariyazono H, Ohno T, Ihara K, Igarashi H, Joh-o K, Ishikawa S, Hara T: Rapid detection of the $22 \mathrm{q} 11.2$ deletion with quantitative real-time PCR. Mol Cell Probes 2001, 15:71-73.

5. Shi YR, Hsieh KS, Wu JY, Lee CC, Tsai CH, Yu MT, Chang JS, Tsai FJ: Genetic analysis of chromosome 22q11.2 in congenital heart disease. J Clin Lab Anal 2003, 17:28-35.

6. Weksberg R, Hughes S, Moldovan L, Bassett AS, Chow EWC, Squire JA: A method for accurate detection of genomic microdeletions using realtime quantitative PCR. BMC Genomics 2005, 6:180

7. Weksberg R, Stachon AC, Squire JA, Moldovan L, Bayani J, Meyn S, Chow E, Bassett AS: Molecular characterization of deletion breakpoints in adults with 22q11 deletion syndrome. Hum Genet 2007, 120:837-845.

8. Gothelf D, Frish A, Michaelovsky E, Weizman A, Shprintzen RJ: Velo-CardioFacial Syndrome. J Ment Health Res Intellect Disabil 2009, 2:149-167.

9. Ben-Shachar S, Ou Z, Shaw CA, Belmont JW, Patel MS, Hummel M, Amato S, Tartaglia N, Berg J, Sutton VR, Lalani SR, Chinault AC, Cheung SW, Lupski JR, Patel A: 22q11.2 distal deletion: a recurrent genomic disorder distinct from DiGeorge syndrome and velocardiofacial syndrome. Am J Hum Genet 2008, 82:214-221

10. NCBI SNPs Database. [http://www.ncbi.nlm.nih.gov/snp].

11. Stevenson J, Hymas W, Hillyard D: Effect of sequence polymorphisms on performance of two real-time PCR assays for detection of Herpes simplex virus. J Clin Microbiol 2005, 43:2391-2398.

12. Taris N, Lang RP, Camara MD: Sequence polymorphism can produce serious artefacts in real-time PCR assays: hard lessons from Pacific oysters. BMC Genomics 2008, 9:234.

13. Rozen S, Skaletsky HJ: Primer3 on the WWW for general users and for biologist programmers. In Bioinformatics Methods and Protocols: Methods in Molecular Biology. Edited by: Krawetz S, Misener S. Totowa, NJ: Humana Press; 2000:365-386.

\section{Pre-publication history}

The pre-publication history for this paper can be accessed here: http://www.biomedcentral.com/1471-2350/12/61/prepub

doi:10.1186/1471-2350-12-61

Cite this article as: Frigerio et al.: SNPs and real-time quantitative PCR method for constitutional allelic copy number determination, the VPREB1 marker case. BMC Medical Genetics 2011 12:61.

\section{Submit your next manuscript to BioMed Central and take full advantage of:}

- Convenient online submission

- Thorough peer review

- No space constraints or color figure charges

- Immediate publication on acceptance

- Inclusion in PubMed, CAS, Scopus and Google Scholar

- Research which is freely available for redistribution

Submit your manuscript at www.biomedcentral.com/submit 